\title{
Industrie 4.0 und die Rolle des CISO
}

Die produzierende Industrie steht derzeit vor großen Herausforderungen, wie etwa der Anpassung von Lieferketten und Wertschöpfungsnetzwerken und der Investition in Innovation und Nachhaltigkeit aufgrund der - bedingt durch die Corona-Krise - negativen Entwicklungen bei Umsatz, Cashflow und Gewinn.

Innovationsdruck in den Unternehmen entsteht insbesondere auch durch den Wandel zur Industrie 4.0, indem intelligente Fabriken das verarbeitende Gewerbe revolutionieren. Die smarte Fertigung generiert hierbei Unmengen an Daten, die dazu dienen, Produktionsprozesse zu verbessern und - zum Teil unter Einsatz künstlicher Intelligenz - Chancen zu erkennen und neue Lösungen zu entwickeln.

Diese wertvollen Daten sind der Grund zur Etablierung innovativer Industrie-4.0-Lösungen und die Basis für Business-Intelligence-Ansätze in den Unternehmen. Sie erlauben neue Erkenntnisse zu gewinnen, validere Prognosen zu erstellen und nachhaltige Entscheidungen zu treffen.

Der Einsatz von digitalen, datenbasierten Technologien erfordert auch einen stärkeren Fokus auf Datensicherheit und Datenschutz. Hierfür etablieren Unternehmen zunehmend die Position eines Chief Information Security Officers (CISO) mit der Aufgabe, die Informationssicherheit zu gewährleisten und damit unter anderem die im Rahmen der intelligenten Produktion gewonnenen Daten zu schützen - diese sind nämlich die Basis des zukünftigen Unternehmenserfolgs im produzierenden Gewerbe.

Ihre ZWF-Herausgeber und-Redaktion

KPM - Die Königliche Porzellan-Manufaktur Berlin

Die Königliche Porzellan-Manufaktur Berlin ist der älteste, produzierende Handwerksbetrieb der Hauptstadt. Hier trifft traditionelles Meisterhandwerk auf modernste Technik. Das von Generation zu Generation überlieferte Wissen zur Fertigung, wird stets weiterentwickelt. So verwandeln die spezifischen Brennvorgänge bei bis zu $1.400{ }^{\circ} \mathrm{C}$ die Rohstoffe Kaolin, Feldspat und Quarz in das edle Material Porzellan. Die dabei entstehende, überschüssige Prozessabwärme wird dank modernster Anlagen nicht verschwendet, sondern in das Berliner
Fernwärmenetz eingespeist. Die KPM Berlin sorgt somit für rund 150.000 heiße Duschen in der Nachbarschaft.

Kontakt:

KPM Königliche Porzellan-Manufaktur

Berlin GmbH

Wegelystraße 1

10623 Berlin

Tel: +4930390090

E-Mail: info@kpm-berlin.com

www.kpm-berlin.com

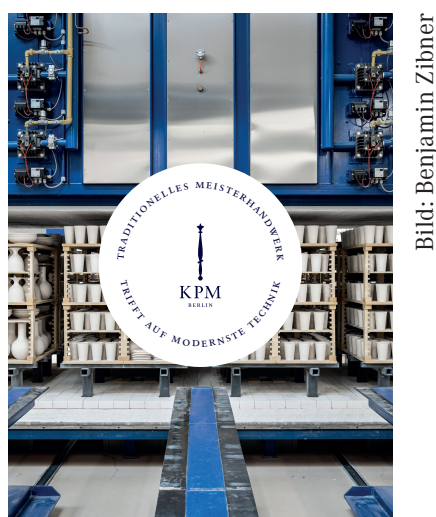

